\title{
Design of problem-based learning activities in the field of microfluidics for 12- to 13-year-old participants-Small Plumbing!: empowering the next generation of microfluidic engineers
}

\author{
H. Bridle ${ }^{1}$ J. Morton ${ }^{2}$ P. Cameron ${ }^{3}$ - M. P. Y. Desmulliez $~ \cdot$ M. Kersaudy-Kerhoas ${ }^{1,5}$
}

Received: 15 April 2016 / Accepted: 22 June 2016 / Published online: 1 July 2016

(C) The Author(s) 2016. This article is published with open access at Springerlink.com

\begin{abstract}
Public engagement activities based on microfluidics are being increasingly delivered and reported on in the literature. Here, we evaluate the success of a novel approach to microfluidics outreach recently undertaken with schoolchildren aged 12-13. Unlike previous work, a problem-based learning approach was adopted whereby participants were asked to design and test a microfluidic system to solve a research challenge. Our aim was to develop understanding of microfluidics design, manufacture and operation via involvement in the full engineering cycle of a product, from ideas to design, and from fabrication to test. This article demonstrates that problem-based learning is a successful method of public engagement with microfluidics, and we share our best practice, including activity design, supporting material produced for the project and an example case study detailing the types of chips
\end{abstract}

Electronic supplementary material The online version of this article (doi:10.1007/s10404-016-1770-x) contains supplementary material, which is available to authorized users.

M. Kersaudy-Kerhoas

m.kersaudy-kerhoas@hw.ac.uk

1 Institute of Biological Chemistry, Biophysics and Bioengineering, Heriot-Watt University, Edinburgh EH14 4AS, UK

2 Institute of Photonics and Quantum Systems, Heriot-Watt University, Edinburgh EH14 4AS, UK

3 Novo Science Ltd., 3 Hatton Mains Cottages, Dalmahoy, Edinburgh EH27 8EB, UK

4 Institute of Signal, Sensors and Systems, Heriot-Watt University, Edinburgh EH14 4AS, UK

5 Division of Infection and Pathway Medicine, University of Edinburgh, 49 Little France Crescent, Edinburgh EH16 4SB, UK produced by the participants. Furthermore, following an evaluation of the activity by all participants recommendations for delivery of this, or similar, activities are provided.

Keywords Public engagement · Microfluidics ·

Schoolchildren $\cdot$ Problem-based learning

\section{Introduction}

Microfluidics is a fast evolving field which presents exciting future perspectives in a wide variety of fields (Sackmann et al. 2014). From fuel cells (Kjeang et al. 2009) to stem cells, from laboratory equipment to point-of-care diagnostics (Chin et al. 2012), both the research and industry communities have been taking advantage of the nature of fluid behaviour at the microscale. Although these advancements have sparked a lot of enthusiasm in the scientific community, they lack wider recognition outside. This is not surprising as the technology has mostly been used so far in high-end specialised products, such as digital polymerase chain reactions or capillary electrophoresis, that the wider public does not have direct contact with, or in systems like pregnancy testing kits where the fluidics aspect is not directly apparent. However, with the rapidly growing number of applications, microfluidics is gradually entering the global market and hence everyday life, for example with the development of point-of-care diagnostics. Therefore, it is timely to raise public awareness of this technology, and perhaps, especially among the young people who will be end-users as more products come to market.

One way to engage a wider audience with microfluidics is through educational and public engagement activities. A wide range of microfluidics-based outreach activities have been designed and delivered, targeting age groups from 
university education through to young children and families at science festivals, an overview of which is given in a recent Biomicrofluidics paper (Esfahani et al. 2016). Some activities demonstrate microfluidic principles by taking a fun "mocked-up" or scaled-up approach using materials like Lego (Jimenez et al. 2015), modelling clay (Jimenez and Bridle 2015), Jell-O (Yang et al. 2010) and chocolate (Esfahani et al. 2016). Others allow participants to use microfluidics to undertake diagnostics or other example experiments illustrating the potential application areas (Hemling et al. 2014). Several low-cost fabrication methods, like shrink film (Nguyen et al. 2011), have been developed to allow for microfluidics education in the school classroom enabling students to fabricate and test devices.

Microfluidics, a field at the interface of chemistry, physics, engineering and life sciences, is an excellent topic for schools outreach offering a platform for interdisciplinary learning thus meeting government objectives. For example, interdisciplinary learning is a key focus in the Scottish High School curriculum, ${ }^{1}$ the "Curriculum for Excellence" whereby interdisciplinary learning is described by the Scottish Government as enabling "teachers and learners to make connections across learning through exploring clear and relevant links across the curriculum", supporting "the use and application of what has been taught and learned in new and different ways" and providing "opportunities for deepening learning, for example through answering big questions, exploring an issue, solving problems or completing a final project". In addition, microfluidic systems are relatively easily operated, using innocuous fluids, either manually or with low-cost syringe pumps. Furthermore, devices are portable and can be made to be very visually appealing, for example, by use of highly coloured food dye solutions.

The majority of microfluidics outreach activities detailed thus far in educational settings are predominantly directive, with students manufacturing pre-determined designs (Fintschenko 2011), and following "recipe-style" operating and testing procedures. Therefore, students are not exposed to the challenges of microfluidics design and miss out on the life cycle of design iterations to reach a working prototype. In contrast to the directive approach, a lot of research has highlighted the benefits of problem-based learning (PBL) approaches (Walker et al. 2015). PBL is a method by which students learn through facilitated problem solving and has been shown to increase confidence and student engagement in laboratory style activities (McDonnell et al. 2007).
This article presents the results of an outreach activity which entices schoolchildren to act as microfluidic engineers to solve a research problem in a team. The advantage of this approach is that the linkages between the science and engineering of microfluidic devices and everyday applications are highlighted for the participants. Importantly, students are challenged to work through the whole design life cycle from specifying the requirements of their device, designing the device, feeding back on manufacturing iterations to finally devising an appropriate testing regime. The detailed work scheme utilised in this project is shown and described, to enable others to replicate this or a similar project. Furthermore, a case study is presented of a device developed by the schoolchildren, which is now being employed in ongoing academic microfluidic research, demonstrating that this type of outreach activity is not just a one way flux of ideas but an opportunity for knowledge exchange. An evaluation of the activity upon all participants, including schoolchildren, teachers and the researchers delivering the project, provides recommendations for future activities of this type.

\section{Description of the activities}

\subsection{Scope of the activity and timeline}

The "Small Plumbing: empowering the next generation of microfluidic engineers" is one of the several microfluidic outreach activities conducted at Heriot-Watt University (Mielczarek et al. 2014; Jimenez and Bridle 2015). This activity was funded by the British Royal Academy of Engineering under the Ingenious Scheme, an awards scheme for projects that engage the public with engineers and engineering (Rios et al. 2012). The short name of the project "Small Plumbing" was inspired by Stephen Quake, a microfluidics academic and entrepreneur, cofounder of the microfluidic company Fluidigm. In a 2009 New York Times Opinion piece, ${ }^{2}$ Stephen Quake described microfluidics as "the technical name for Small Plumbing" and argued that practical problems can be equally important as fundamental ones, enticing engineers and scientists to work across the traditional divide of pure and applied sciences.

The project was specifically designed to deliver an introduction to microfluidics to schoolchildren aged 12-13 and provide an outreach experience to university student scientists and engineers. The overarching goal, as specified by

\footnotetext{
2 http://www.nytimes.com/2009/02/18/opinion/18iht-edquake.1.

20274600.html?_r=1.
}

\footnotetext{
1 http://www.educationscotland.gov.uk/learningandteaching/learningacrossthecurriculum/interdisciplinarylearning/index.asp.
} 
Fig. 1 a Timeline of the overall project. b Diagram of the microfluidic outreach programme work flow. Fabrication and test of the microfluidic chips were carried out at HeriotWatt University prior to the final school session. This program intended to show students the full engineering cycle of a product

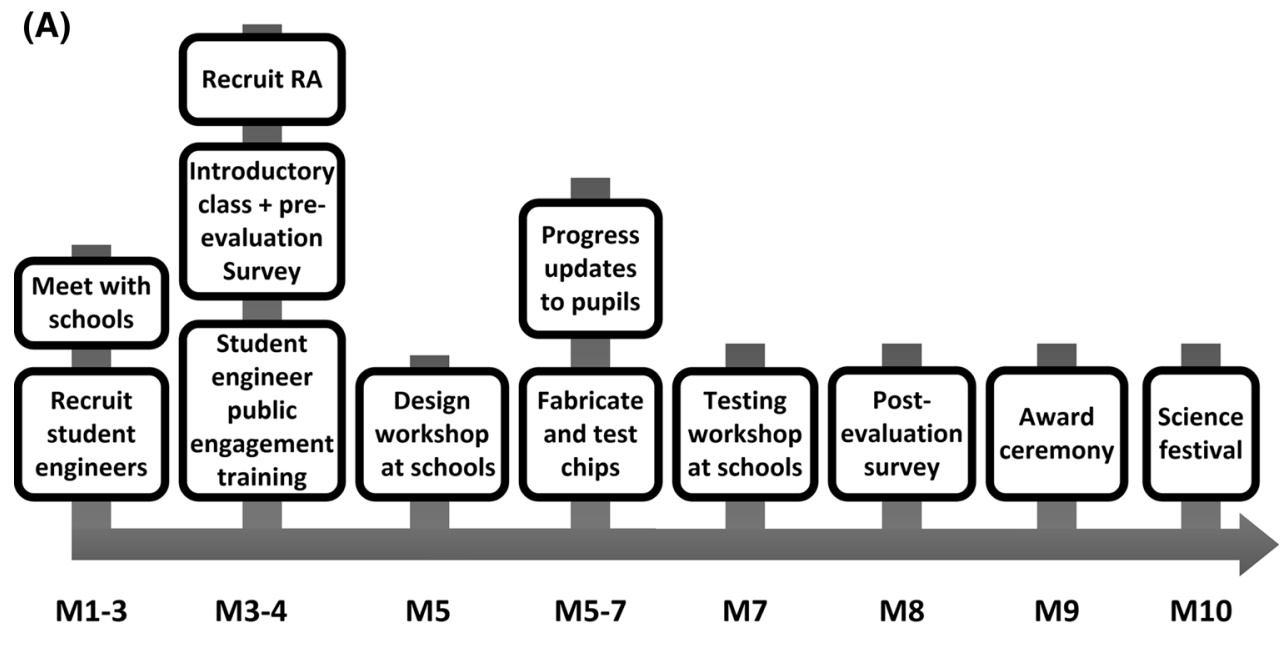

(B)

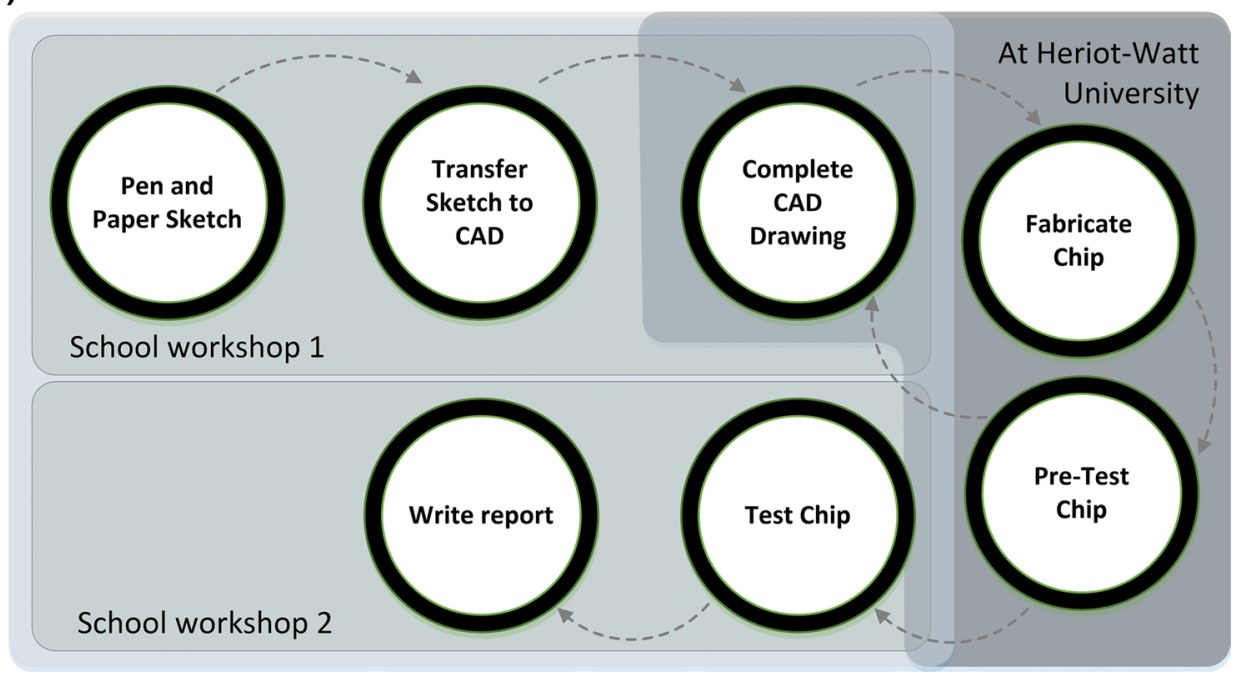

the scheme, was to promote engineering and to provide outreach training and experience to university mentors. The detailed learning objectives were: (1) Inspire a future generation of engineering practitioners and researchers to become involved in microfluidic engineering, and more generally the field of engineering, in order to tackle biological, medical or environmental problems; (2) develop expertise and skills of researchers in the field of public engagement, enabling them to successfully contribute to public engagement events; and (3) enable pupils to discuss engineering and its impact on their daily lives.

An additional aim was to assess the feasibility and success of PBL as an approach to microfluidics outreach education. As far as the schoolchildren were concerned, the key learning outcomes targeted were: (1) to gain an understanding of the basic principles of microfluidics and laminar flow; (2) to acquire knowledge of the different components which could be incorporated on-chip, e.g. mixers, gradient generators etc.; and (3) to develop the ability to design and operate microfluidic systems.
An overview of the programme of activities is presented in a timeline shown in Fig. 1a. An introductory lecture was given by two academics (Dr. Bridle and Dr. Kersaudy-Kerhoas) followed by two teamwork sessions in the schools. In the first session, each team of schoolchildren met their mentor, who described a research challenge, typically related to their own work and together the team brainstormed a microfluidic solution delivering a pen and paper annotation of their microfluidic chip as well as a computer-aided design (CAD) drawing. The deliverable of the first session was a microfluidic design on paper and a basic CAD design. A dedicated postdoctoral research associate (Dr Morton) then completed the CAD drawings and fabricated 14 working chips over a period of 3 months. During this 3-month period, a set of three project updates, spaced out by 4 weeks, were delivered to the school pupils. These updates provided written and visual guidance on the progress of the fabrication of their chips and allowed students an opportunity to feed into the iterative design process by responding and asking questions directly to the research 
associate. The updates included any small changes to the designs that were required in order to overcome challenges encountered in the fabrication process. Some of the schoolchildren had the opportunity to visit the university and participate in the fabrication process.

In the second session, the pupil teams tested the devices and prepared a report describing the chip they had designed and tested. The high school teachers organised a follow-up session to finalise the reports before sending them back to the university project team. A small judging panel consisting of two academics and two industrial representatives read and judged the reports prior to an award ceremony. Two pupils involved in the winning projects joined the Heriot-Watt microfluidics stand at the Glasgow Science Festival to present their winning chips.

Work in this project was intended to develop the pupils' appreciation of the engineering design cycle through the development of a microfluidic prototype. In particular, the aim was for the pupils to gain an understanding of the iterative process of CAD design and prototyping, through the descriptions provided within their monthly updates. The use of CAD in relation to microfluidics education was explored in the classroom before (Nguyen et al. 2011) but not with the design freedom involved in this project. Figure $1 b$ summarises the engineering design cycle during this project.

\subsection{Public targeted}

The project engaged with two different state funded schools, reaching a total of 9812 - to 13-year-old pupils in Scotland. The two schools were recruited following a small advertisement campaign by email, which received 13 notes of interest. Each school cohort was divided into teams comprising between 3 and 8 pupils depending on the cohort size in each school. Each pupil team was assigned 1 or 2 university mentors.

\subsection{Mentors}

Mentors were Ph.D. students, postdoctoral researchers or staff members from Heriot-Watt University or the University of Edinburgh, who joined the programme after it was advertised throughout both universities. The mentor team comprised 12 researchers with bioengineering, biology and engineering backgrounds. They were provided with three training sessions prior to the first school sessions. These sessions are described in more detail in a separate evaluation publication (submitted to the Journal of Pre-college Engineering Education Research) investigating the impact of this project, considering gender, on the science, engineering, technology and mathematics (STEM) interest of the schoolchildren. The project thus provided the mentors with outreach training as well as two intensive school workshop experiences.

\subsection{Detailed lesson plan}

Prior to two workshops where the schoolchildren undertook the project, an introductory one-hour class was held to give an overview of the engineering life cycle and introduce the basics and application of microfluidics during a one-hour lesson. The first workshop lasted $90 \mathrm{~min}$ and focused on the teams producing a microfluidic design to solve a problem introduced by their mentor. Each team was provided with a workbook "The Engineer's Notebook" (Suppl. Info. 1), which guided them through the design task and provided the key information on microfluidic design and fabrication that was presented in the first workshop. The pupils were first asked to discuss within their teams for 5 min the concepts of microfluidics that they had learned in the first session. Engineering roles were then divided between the team members (project engineer, mechanical engineer, test engineer and support engineer). This was designed to demonstrate the various roles that an engineer can have within a team, the skills related to these roles. The groups were then asked to define the specific problem introduced by their mentor. A series of questions laid out in the workbook helped to guide their thinking process. The pupil teams were then asked to brainstorm microfluidic solutions to their problem and come up with the key design requirements. They were asked to consider the materials needed to make their microfluidic chips, from a choice of paper or plastic, where the key properties of each material were provided. The pupils had to decide on the specific processes (inlets and outlets, mixing, gradient generation, delays) that their chips had to include. To support the pupil teams in this task, a series of microfluidics component cards were produced providing the teams with an active hands-on way to visually put together the series of actions necessary to solve their challenge (cards shown in Suppl. Info. 1, pp. 15-17) and brainstorm ideas. The design drafted by the pupil on paper was transformed into a CAD drawing by some of the pupils, while others planned the test protocol for the second session, with the help of their mentor(s). Finally, the teams were asked to consider the safety requirements that their project implied and to discuss how their chip would be fabricated and tested.

In the second workshop, also $90 \mathrm{~min}$ in total, each group had 20 min of catch-up time, discussing the fabrication of the chip and its goal, followed by $30 \mathrm{~min}$ of experimental time, including setting up the experiments, putting on safety equipment and taking measurements. In order for the pupils to appreciate the range of microfluidic solutions achieved during this project, as well as to practice the 
Table 1 Lesson plans for (A) the first workshop whose deliverable was a sketch of the chip and a CAD file in a. dxf format and (B) the second workshop whose deliverable was a report

A. First Workshop-Total 1 h 30 min

Group introduction, team member roles and recapitulation of introductory lesson

Problem and solution requirements

Processes (cards)

Technical drawing

Experiment design, safety requirement and final discussion

B. Second workshop — total time 1 h $30 \mathrm{~min}$ Catch-up time

Set-up time experiments

Reporting
Group introduction. Mentors were asked to bring props to explain their research in an accessible way to 12- to 13-year-old pupils

Recapitulation of the techniques/building blocks/material seen in the introductory lesson

Presentation and choice of different engineering roles

Discussion of the problem

Discussion on the manufacturing aspects

Establish chip requirements

Choice of process modules using card sets

Drawing of the microfluidic network on paper

Drawing of the microfluidic network on CAD, design of the test experiment and risk assessment

Discussion on report + any questions

Recapitulation of the project by organisers

Reintroduction of the teams and mentors

Recapitulation of the manufacturing process and the changes made to the design (Progress reports)

Presentation of the test procedures and the report. Set-up the microfluidic chip

Recapitulation of safety procedures if any

Protective equipment (goggles, laboratory coats and gloves)

Test procedures, measurements, photographs

Opportunity to circulate and view the work of others; chance for the pupils to present their chips to their peers

Back to the report and work on all the sections. Some pupils can draw pictures, while others write parts of the report
$10 \min$

$20 \min$

$5 \min$

5 min whistle-move to next stage

$5 \mathrm{~min}$

$15 \min$

$5 \mathrm{~min}$

5 min

10 min whistle-move to next stage

$10 \mathrm{~min}$

$15 \mathrm{~min}$ whistle-move to next stage $15 \mathrm{~min}$

$20 \mathrm{~min} 25 \mathrm{~min}$

$5 \min$

$10 \mathrm{~min}$

$20 \min$

5 min

5 min whistle-move to next stage

$\sim 10 \mathrm{~min}$

$30 \min$

$\sim 5 \min$

$\sim 5 \mathrm{~min}$

$\sim 10$ min whistle - move to next stage

$\sim 10$ min whistle-move to next stage $30 \mathrm{~min}$

$\sim 20 \min$ description and demonstration of their own work, $10 \mathrm{~min}$ was dedicated to pupils circulating and observing demonstrations of the other microfluidic systems. This was followed by 20 min to write up their results. The lesson plans are described in Table 1.

\subsection{Example projects}

A selection of projects developed by the pupils is presented in Table 2. The projects introduced pupils to a range of different microfluidic components. Table 2 shows how the other more specific concepts, such as the formation of droplets, flow rate-driven flow or magnetic separation, were particular to the individual projects.

\section{Materials and methods}

\subsection{Support material for mentors, teachers and pupils}

A total of 4 handbooks were produced for the project; these are provided in Supplementary Information section. The Handbook for University Participants guided the mentors through the project goals, basics of microfluidics and a series of questions to help them to come up with a mini research project for their school pupil team (Suppl. Info. 2). A teacher's reference book provided the teachers with notes regarding the project, including a presentation of the team members, basics of microfluidics, a glossary and a description of the activities along with their fit to the Curriculum 
Table 2 Example projects are grouped by topic, briefly described linked to learning objectives

\begin{tabular}{|c|c|c|c|}
\hline Topic & $\begin{array}{l}\text { Name of the project and description of the } \\
\text { chip }\end{array}$ & Photos & Learning objectives \\
\hline \multirow[t]{2}{*}{ Art } & $\begin{array}{l}\text { "Lalineic": A chip representing a cartoon } \\
\text { using microfluidic channels }\end{array}$ & & $\begin{array}{l}\text { Visualisation of laminar flow } \\
\text { Formation of droplets in two-phase systems } \\
\text { Applications of microfluidics to art }\end{array}$ \\
\hline & $\begin{array}{l}\text { "PixelSnail": A chip representing a car- } \\
\text { toon using microfluidic channels }\end{array}$ & & $\begin{array}{l}\text { Visualisation of laminar Flow } \\
\text { Concepts of flow rate-driven flows and } \\
\text { micromixing } \\
\text { Applications of microfluidics to art }\end{array}$ \\
\hline \multirow[t]{2}{*}{ Environment and chemistry } & $\begin{array}{l}\text { "H2Know": A chip with integrated pH } \\
\text { measurements of water samples }\end{array}$ & & $\begin{array}{l}\text { On-chip filtration } \\
\text { PH measurements }\end{array}$ \\
\hline & $\begin{array}{l}\text { "ScrumpleFrumps": A chip enabling the } \\
\text { trapping of oceanic micro-organisms, } \\
\text { inspired by the work of Bouchillon et al. } \\
(2014)\end{array}$ & & $\begin{array}{l}\text { Basics of diffusion } \\
\text { Basics of photolithography } \\
\text { Applications of microfluidics to environ- } \\
\text { mental sciences }\end{array}$ \\
\hline \multirow[t]{3}{*}{ Life sciences } & $\begin{array}{l}\text { "pH droppers": A chip capable of encap- } \\
\text { sulating biological probes into polymer } \\
\text { droplets }\end{array}$ & & $\begin{array}{l}\text { Visualisation of laminar Flow } \\
\text { Droplet microfluidics } \\
\text { Applications of microfluidics to diagnostics }\end{array}$ \\
\hline & $\begin{array}{l}\text { "DJ Cal Cal": A chip creating a gradient of } \\
\text { Irn-Bru solution in water, to test cellular } \\
\text { response }\end{array}$ & & $\begin{array}{l}\text { Visualisation of laminar Flow } \\
\text { Droplet microfluidics } \\
\text { Applications of microfluidics to Biological } \\
\text { sciences }\end{array}$ \\
\hline & $\begin{array}{l}\text { "MLE": Using magnetic fields to sort } \\
\text { blood cells }\end{array}$ & & $\begin{array}{l}\text { Visualisation of laminar Flow } \\
\text { Basics of magnetic fields and how to use } \\
\text { them to sort cells } \\
\text { Applications of microfluidics to diagnostics }\end{array}$ \\
\hline
\end{tabular}

CAD files are available upon request

of Excellence (Suppl. Info. 3). Two handbooks were produced for the pupil teams, one for each of the workshop: The Engineer's Notebook (Suppl. Info. 1) and The Engineer's Scientific Report (Suppl. info. 4). The Engineer's Notebook guided the pupils and their mentor through a series of milestones during the 80-90-min session. During the session, a whistle was blown at key time intervals to suggest to the teams that they should move onto the next section. This meant that mentors themselves did not have to keep track of time and could focus on engaging with the students. The project workbooks also helped to keep a written trace of the work carried out in each session. 


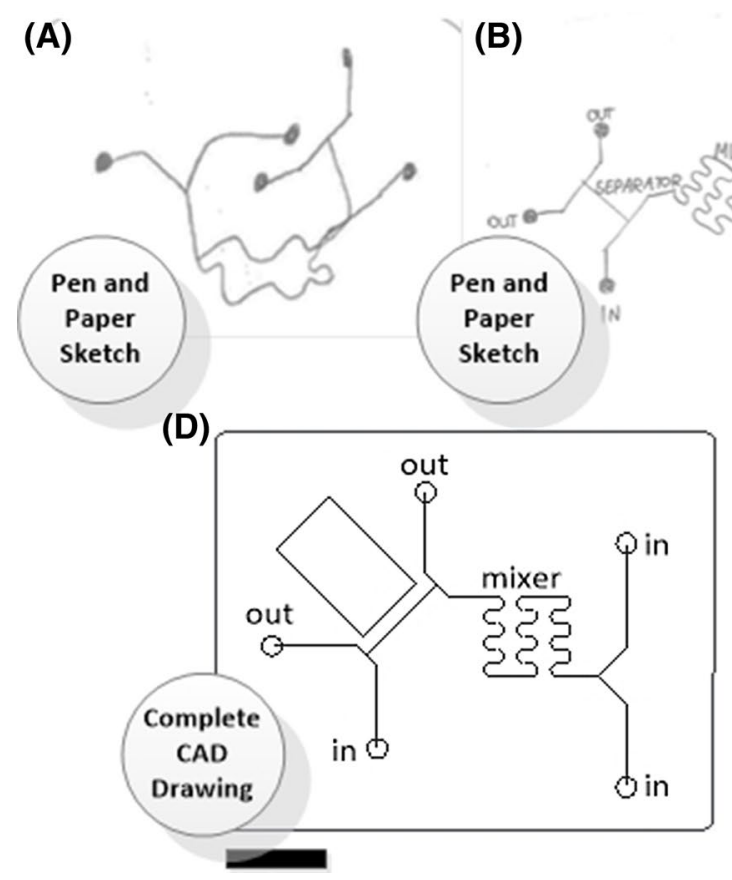

Fig. 2 Engineering cycle of a microfluidic chip illustrated. a First sketch of the "MLE" chip (c.f. Table 2) produced by the pupils, $\mathbf{b}$ final sketch attempt by the pupils, $\mathbf{c}$ CAD produced by the pupils on

\subsection{Manufacture of microfluidic devices}

Computer-aided design (CAD) drawings on a 2D drawing CAD software (AutoCAD, Autodesk, UK, free student version) were produced by the schoolchildren during the activities. These drawings were then inspected and completed at Heriot-Watt University. As part of the design requirements, pupils used channels with widths between 200 and $500 \mu \mathrm{m}$, to be compatible with the rapid prototyping technique developed in-house. Briefly, this technique involves the solvent (ethanol)-assisted thermal bonding of thin sheets of PMMA under heat $\left(70{ }^{\circ} \mathrm{C}\right)$ and pressure $(1.6 \mathrm{MPa})$. For the majority of the devices, the base and top layers were prepared using 2.0-mm-thick sheets of PMMA, while the intermediate channel layers were prepared using 0.5-mm-thick PMMA, cut to size using a laser cutter (Epilog Mini 18, 40 W, Epilog, $\mathrm{CO}$, USA). One of the pupils designs required dimensions smaller than $200 \mu \mathrm{m}$ to function and was therefore manufactured using a standard soft lithography method using Polydimethylsiloxane (PDMS) cast on an SU8 mould as described in (Jenkins 2013). The PDMS was peeled off its mould, inlet holes were punched using a biopsy tool and PDMS slabs simply positioned on a clean glass wafer for operation.

\subsection{Operation of microfluidic devices}

Both custom-made as well as standard luer-lock connectors and 1/16" ID ETFE tubing (VWR, UK) were used to

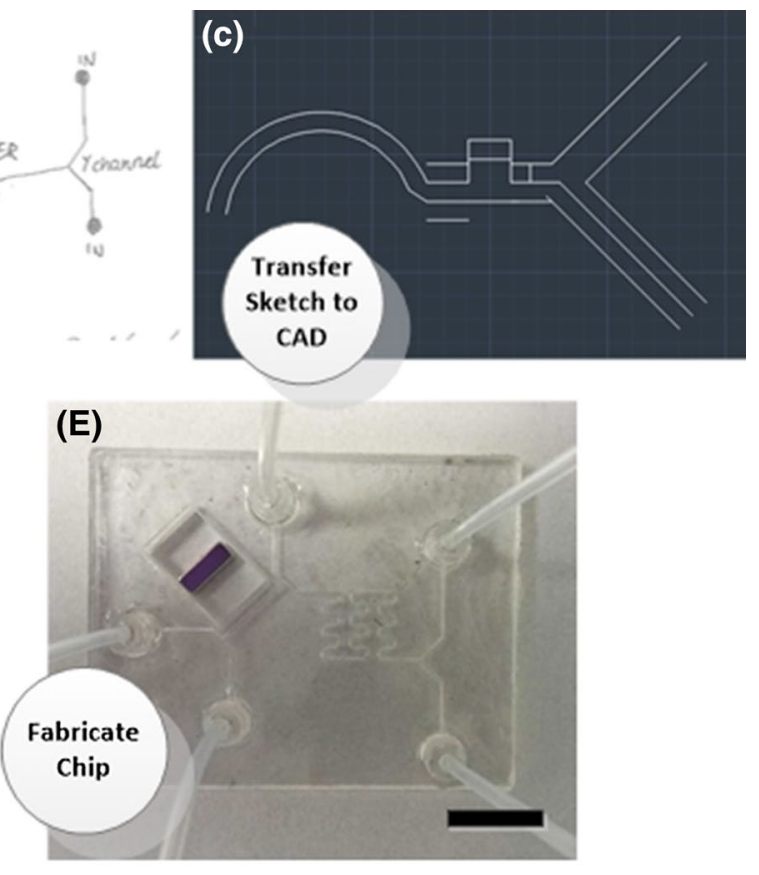

the first workshop, $\mathbf{d}$ final CAD produced by Heriot-Watt; scale bar is $1 \mathrm{~cm}$, and e photograph of the chip prototype. Scale bar is $1 \mathrm{~cm}$

connect syringes to the chips. Flow into the devices was actuated manually by the pupils or by using syringe pumps (Aladdin range, WPI, FL, USA).

\subsection{Safety}

Schoolchildren were encouraged to develop testing protocols using innocuous chemicals such as food dyes. Food dyes were obtained in a variety of colours from Classikool. Other reagents were necessary for particular projects including Universal Indicator and Bromocresol Purple (Sigma-Aldrich).

\subsection{Evaluation}

In collaboration with the Royal Academy of Engineering and Novoscience Ltd, an evaluation was undertaken. The evaluation technique and results are presented in details within another publication focussing on the use of this type of project to engage pupils with STEM topics and particularly encourage the participation of females into STEM subjects and careers. Here, the answers to three questions from the evaluation data are discussed, focussing on the basic rating and experience of the activity by all participants, and specifically, the schoolchildren were asked whether they liked the activities and to rate them against a set number of adjectives; the teachers were asked an open question about their opinion of the project; and the mentors 
were asked to rate the impact of the project on their public engagement abilities against a set of provided criteria and also a more open-ended question about their experiences. Here, this data is utilised to assess the success of the PBL approach to microfluidics outreach and provide recommendations for future such projects.

\section{Results and discussion}

This project intended to show the pupils the full engineering cycle needed to design, manufacture and test a product, which, in this case, was a microfluidic chip. Unlike many other microfluidic outreach workshops for schools or science exhibitions, the project took a novel approach allowing pupils design freedom in response to a challenge set by their mentors. This allowed pupils to develop their own ideas, take part in the design of the chip and control over how to test the chips met the original design requirements. Since there were just two school workshops of about $90 \mathrm{~min}$ each, university mentors were asked to come up with an idea for a problem-based learning challenge prior to the first workshop and find ways to engage the pupils with their initial idea. During the first workshop, pupil teams were introduced to their mentor(s). They discussed the research subject brought by the mentor(s) and started sketching ideas on papers, using the component cards to structure designs. After $40 \mathrm{~min}$, they were asked to start transferring their sketches onto a CAD Software, in this case, AutoCAD 2014 (free student version) or CorelDraw. The task appeared daunting to some students, but encouraged by their mentors, even the most apprehensive pupils enjoyed learning how to draw their sketches on computers. Previous PBL evaluation indicated that significant demonstrator involvement and management of the facilitated learning are key for a successful outcome (Kirschner et al. 2006), and this is particularly essential in this type of outreach activity with limited time and a challenging task. In Fig. 2, the 4 stages of this engineering cycle are presented for a magnetic separation chip as experienced by the pupils: Fig. 2a, b-sketches as drawn by pupils; Fig. 2cfirst CAD drawing by the pupils; Fig. 2d-final CAD produced at Heriot-Watt and shared with the pupil team; and Fig. 2e-first chip prototype ready for test at school.

\subsection{Chips developed and associated learning objectives}

The detailed learning objectives for the schoolchildren were to inspire pupils to become involved in engineering and to enable the pupils to discuss the impact of the project, and engineering in general, on their daily lives. All reports produced by the pupils provided evidence of understanding of the wider utilisation of their system and why this was important. This evidence was further reinforced by observation of the pupils at the awards ceremony, and the science festival, as they explained their work, and its wider significance, to their parents and the general public.

Despite no prior knowledge of microfluidics before the project, all teams developed a working prototype. Overall, fourteen successful projects were delivered across a variety of topics, organised into three groups: arts, environmental and medical applications. Example projects in environmental and medical application groups included making a miniaturised lemon battery, testing for counterfeit drugs, analysing water quality and separating blood cells for medical therapies (c.f. Table 2). Several projects focused on the theme of art, which were included as a means of engaging those pupils with a strong interest in artistic creativity and design. Microfluidics lends itself well to artistic projects as microchannels filled with coloured dyes can be visually clear and attractive. Microfluidic "art" has been promoted by other groups as a means of science outreach itself (Cleary et al. 2008).

The key microfluidics learning outcomes were met by all groups with the workbooks demonstrating an understanding of the basics of microfluidics including laminar flow and Reynolds number (outcome i). Every group successfully designed and operated a microfluidic device (outcome iii) solving their research challenge. The opportunity to circulate at the end of the testing workshop ensured that all schoolchildren saw a variety of microfluidics components in action (outcome ii) as well as developing deeper understanding of the components needed for their particular system.

Each project met the key microfluidics learning outcome of understanding, and allowing the visualisation of, laminar flow. Furthermore, each project met the additional aims (discussed at the end of Sect. 2.1) relating to demonstrating the concept of the engineering design cycle and CAD design. One award-winning project will be presented in further detail as a case study. Posters created for the award ceremony and describing the microfluidic chips, aimed at the young public and their parents, are presented in Supplementary Information (Suppl. Info. 5).

\subsection{Case study: a droplet generation chip}

This case study was inspired by a current real-life scientific challenge within a project entitled PROTEUS and funded by the Engineering and Physical Research Council (EPSRC), one of the major research funding agencies in the UK. ${ }^{3}$ The project brings together a team of scientists who are using photonic-based solutions to diagnose infection, inflammation or scarring in patients' lungs. The novel

\footnotetext{
3 http://gow.epsrc.ac.uk/NGBOViewGrant.aspx?GrantRef=EP/ K03197X/1.
} 
Fig. 3 pH droppers microfluidic droplet chip. a Schematic of the chip. The scale bar represents $1 \mathrm{~cm}$. b Concept for the formation of aqueous droplets in a mineral oil solution. This drawing is not to scale, $\mathbf{c}$ Photograph of the chip manufactured. The yellow arrow represents the polymer (yellow dye) flow, the blue arrow the probe solution (blue dye) flow, the black arrow the mineral oil flow and the green arrow the output aqueous droplet flow in the mineral oil. d Experimental data obtained by the pupils measuring the droplet length versus the oil flow rate. A.U. arbitrary unit as the microscope was not calibrated (colour figure online)

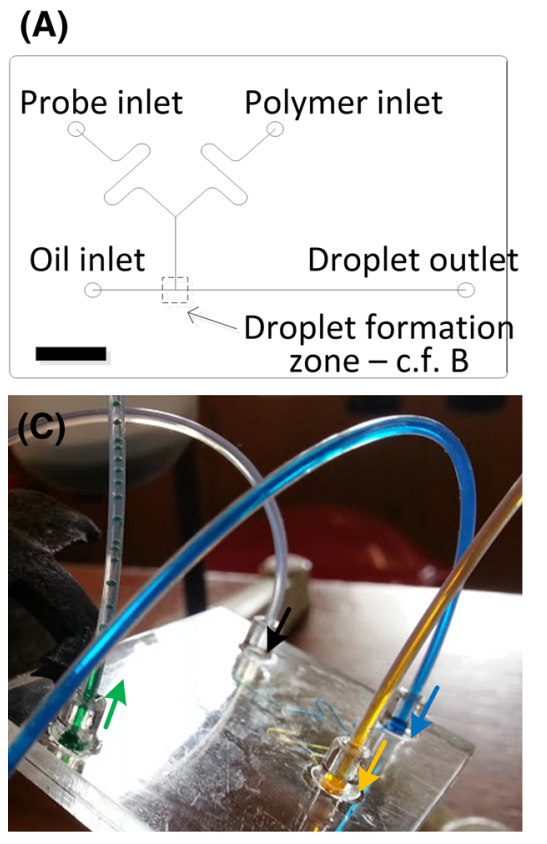

(B)

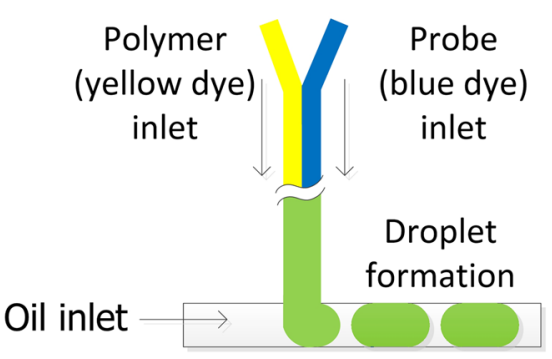

(D)

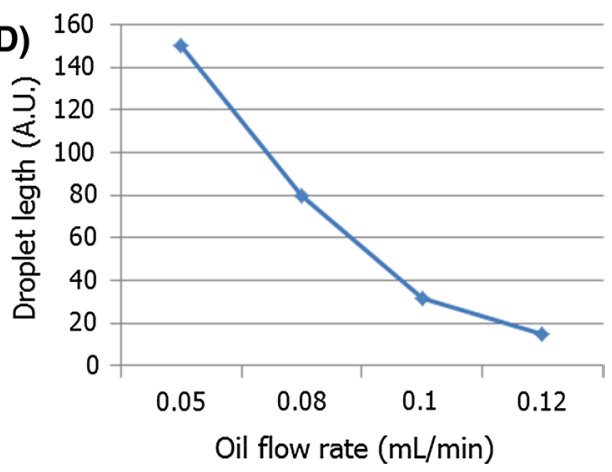

molecular imaging technique consists of fitting chemical sensors to the end of optical fibres. The problem presented to the pupils was to research ways to encapsulate the nanosized sensors in larger shells. The encapsulation was necessary to enable effective immobilisation of the sensors at the end of the optical fibres. The team of pupils working on this project designed a chip consisting of three inlets comprising the sensor solutions represented by a yellow dye, a polymeric solution represented by a blue dye and a mineral oil solution as shown in Fig. 3a. The chip was manufactured as described in Material and Method section. Three syringe pumps were necessary to actuate the flow at various rates. After developing a fundamental understanding of laminar flow through initial observation of their fabricated chip, they discussed the influence of the water-based and oil-based flow rate ratios on the size of the droplets. Based on image analysis, pupils measured the size of the droplets versus the oil flow rate (Fig. 3b). In Supplementary Information, the reader can find a video of droplets produced by this chip (Suppl. Info. 6). This case study was selected for presentation not only because the schoolchildren won a prize for their work at the awards ceremony, but also as it is an excellent example illustrating knowledge exchange, i.e. that the outreach was not a one-way flow of ideas but that the work of activity participants, in this case, the schoolchildren can feedback into academic research. The microfluidic devices produced in this project have been delivered to researchers working on the PROTEUS project to evaluate and for solving the encapsulation issue. ${ }^{4}$

\footnotetext{
${ }^{4}$ http://www.edinburghnews.scotsman.com/news/education/pupilsdesign-microchip-to-fight-lung-disease-1-3816614.
}

\subsection{Project follow-up and pupils engagement}

Projects updates were delivered to the school pupils every 4 weeks, for the 12-week duration of the fabrication process. These project updates consisted of a 3-4-page document in a.pdf format with the developments of the CAD drawings of their team's chip in the first 1-2 updates and annotated photographs of the fabricated prototypes in the last two updates. Questions for reflection were added at the end of each update to allow the pupils to consider the progress and any alterations made, as well as providing them with a method of feeding back to the Research Associate manufacturing their devices. This represents a unique aspect of this project giving pupils insight into the iterative process of microfluidics design and manufacture. The pupils' responses to these questions reflected their interest in their project. Several teams asked about how long it would take to make the chips, others asked how it would benefit them in the future, or would it be "commonly used" afterwards. One team asked about how much the chip would cost. Some of these quotes are listed in Suppl. Info. 7. All these considerations show that the pupils are very keen on the relevance of this type of project (Esfahani et al. 2016). Towards the 3rd month, a team from one of the school, accompanied by one of their teachers, came to visit our mechanical workshops and participate in the fabrication of the microfluidic chips. All participants, pupils, mentors and teachers, were surveyed before, and after, delivery of the project in order to assess their opinion of the activity. All of this data were combined to produce recommendations for future delivery of these, or similar, activities. 
The impact of the Small Plumbing Project on the knowledge of microfluidics was substantial. All pupil groups delivered a successful design and were able to satisfactorily evaluate the performance of the device during the testing session, indicating that, despite microfluidics being a new concept to the majority of pupils (only two pupils surveyed had heard of microfluidics prior to the project), it is possible to engage them in this kind of problem-based learning approach with microfluidics. From the questionnaire, $84 \%$ of pupils reported that they liked the activities, similar to the reported satisfaction with short workshops of a more traditional science festival outreach nature (Esfahani et al. 2016). However, the advantage of the PBL approach was allowing the schoolchildren the opportunity for deeper learning of microfluidics and greater ownership of the projects/activities. Observation of the groups showed high levels of student engagement and several examples of excitement among students when they worked out how to put together a design to solve the challenge. From a question asking the pupils to assess the project in terms of a number of adjectives, the data showed that a minority of girls (but no boys) felt that the activities were "boring". However, the individuals who assessed the activities as "not for them" reported that they were interested in other subjects but had still found the project "enjoyable", "thought-provoking" and "different".

\subsection{Experience and views of the teachers and mentors}

The teachers were also asked for their opinion of the project. They thought that the approach was a good opportunity for the pupils to do something different and that they had demonstrated a high level of engagement and interest throughout. Additionally, they stated that the possibility to interact with role models was particularly beneficial, an unexpected bonus of this type of PBL approach. The mentors reported increased confidence and interest in public engagement, meeting therefore one of the detailed learning objectives, but commented that the project was especially time-consuming. Some were particularly positive about how the project had generated new research ideas to help them in their day-to-day work, as illustrated in the case study. One specific recommendation, arising from the open-ended question the mentors were asked about that project, was that smaller group sizes were recommended to ensure all pupils could be directly involved in design and experimentation where groups had up to eight pupils not all of them could be involved in setting up and operating the device for example.

\subsection{Recommendations}

While a problem-based learning approach can be a successful means of introducing schoolchildren to microfluidics, there are several recommendations essential to the delivery of such activities:

1. Well-trained mentors to facilitate the PBL process are critical, as detailed in previous literature (Perrenet et al. 2000; Kirschner et al. 2006). This is particularly key in this type of outreach activity introducing a totally new concept to schoolchildren in a short timeframe.

2. Careful planning of the projects to ensure design, manufacture and operational feasibility is essential: Mentors must be involved at this stage.

3. Appointment of a Research Associate to oversee the project and manage communications provides an excellent means to reduce academic workload and ensure successful project delivery.

4. Small group sizes (3-4 pupils) work best for microfluidics outreach of this type allowing all pupils to participate in a hands-on way.

5. Although PBL offers a novel approach and design freedom, such activities must be tightly supervised especially for this age group and time allowance: Workbooks such as those shown in Suppl. Info. 1 and 4 provide an excellent means to guide group progress. Longer, more open-ended activities could be undertaken with more time available.

\section{Conclusions and implications}

This article has demonstrated that allowing schoolchildren the opportunity to fully engage in the design process is a successful approach for introducing schoolchildren to microfluidics. This works best for a project, which can be spread over a period of a few weeks or months, to allow time for manufacturing iterations, and requires considerable input from the project coordinators. In our case, a dedicated Research Associate was employed to manage device fabrication and communication with the schools and we recommend costs involved in this particular resource be requested when planning, and securing support for, this type of project.

We have shared our best practice in delivering this type of project introducing schoolchildren to microfluidics with sufficient information for the project to be replicated elsewhere. We encourage others to consider this full design cycle approach as our evaluation, indicated clear benefits to all participants. For example, the pupils developed a good understanding of the research process in both design and evaluation of their devices and were highly engaged in the activity. Furthermore, the opportunity for interaction with university researchers who can act as role models was a key highlight of this project for the pupils. An additional benefit is that, by linking with existing research, this is not only 
highly relevant for the pupils, but the devices designed in the project can potentially be useful for future activities, as demonstrated in the case study, so although time-consuming for mentors, there is a potential benefit to their research.

Acknowledgments Dr. Maïwenn Kersaudy-Kerhoas, a Royal Academy of Engineering Research Fellow, and all the authors of this article thank the Royal Academy of Engineering Ingenious Scheme for funding the project and the EPSRC funded Impact Acceleration Award at Heriot-Watt University for providing match-funding. In addition, we would like to thank the teachers, headmasters and pupils at Liberton High School and Inverkeithing High School for their participation in the project. We would like to acknowledge support of the Heriot-Watt Engage team Dr. L. Wicks and Dr. K. Przycybien and all our volunteer mentors: A. Graczyk, Dr. A. Kerrouche, A. Liga, Dr. D. Watson, Dr. H. Szoor-McElhinney, J. McGrath, J. Faeber, Dr M. Jimenez, N. Goddard, Dr. P. Pavli, P. Scanlan, R. Saleeb, V. Busin and W. Mielczarek. We acknowledge W. Mielczarek for contribution to the introduction. We would also like to acknowledge the Heriot-Watt Principal's Prize for Public Engagement Team Award, which has allowed us to celebrate the successes of this project and disseminate the results at conferences and through this publication. This outreach activity also helped Dr. Helen Bridle to become the recipient of the Royal Society of Edinburgh Public Engagement Prize 2016.

Open Access This article is distributed under the terms of the Creative Commons Attribution 4.0 International License (http://creativecommons.org/licenses/by/4.0/), which permits unrestricted use, distribution, and reproduction in any medium, provided you give appropriate credit to the original author(s) and the source, provide a link to the Creative Commons license, and indicate if changes were made.

\section{References}

Bouchillon GM, Chau JF, McManus GB, Shor LM (2014) Microfluidic passive samplers for in situ collection of live aquatic protists. Anal Methods 6(20):8350-8357

Chin CD, Linder V, Sia SK (2012) Commercialization of microfluidic point-of-care diagnostic devices. Lab Chip 12(12):2118-2134

Cleary J, Slater C, McGraw C, Diamond D (2008) An autonomous microfluidic sensor for phosphate: on-site analysis of treated wastewater. IEEE Sens J 8(5):508-515

Esfahani MMN, Tarn MD, Choudhury TA, Hewitt LC, Mayo AJ, Rubin TA, Waller MR, Christensen MG, Dawson A, Pamme N (2016) Lab-on-a-chip workshop activities for secondary school students. Biomicrofluidics 10(1):011301
Fintschenko Y (2011) Education: a modular approach to microfluidics in the teaching laboratory. Lab Chip 11(20):3394-3400

Hemling M, Crooks JA, Oliver PM, Brenner K, Gilbertson J, Lisensky GC, Weibel DB (2014) Microfluidics for high school chemistry students. J Chem Educ 91(1):112-115

Jenkins G (2013) Rapid prototyping of PDMS devices using SU-8 lithography. In: Jenkins G, Mansfield DC (eds) Microfluidic diagnostics: methods and protocols. Totowa, NJ, Humana Press, $153-168$

Jimenez M, Bridle HL (2015) Angry pathogens, how to get rid of them: introducing microfluidics for waterborne pathogen separation to children. Lab Chip 15(4):947-957

Kirschner PA, Sweller J, Clark RE (2006) Why minimal guidance during instruction does not work: an analysis of the failure of constructivist, discovery, problem-based, experiential, and inquiry-based teaching. Educ Psychol 41(2):75-86

Kjeang E, Djilali N, Sinton D (2009) Microfluidic fuel cells: a review. J Power Sources 186(2):353-369

McDonnell C, O'Connor C, Seery MK (2007) Developing practical chemistry skills by means of student-driven problem based learning mini-projects. Chem Educ Res Pract 8(2):130-139

Mielczarek W, Aspray TJ, Mallevre F, Jimenez M, McGrath J, Cameron P, Bridle H, Kersaudy-Kerhoas M (2014) Microworld and microflows: initiating school children to microfluidics. In: 4th European conference on microfluidics (IFlu'14). Limerick, Ireland

Nguyen D, McLane J, Lew V, Pegan J, Khine M (2011) Shrink-film microfluidic education modules: complete devices within minutes. Biomicrofluidics 5(2):022209

Perrenet JC, Bouhuijs PAJ, Smits JGMM (2000) The suitability of problem-based learning for engineering education: theory and practice. Teach High Educ 5(3):345-358

Rios A, Zougagh M, Avila M (2012) Miniaturization through lab-ona-chip: utopia or reality for routine laboratories? A review. Anal Chim Acta 740:1-11

Sackmann EK, Fulton AL, Beebe DJ (2014) The present and future role of microfluidics in biomedical research. Nature 507(7491):181-189

Walker A, Leary H, Hmelo-Silver CE, Ertmer PA (2015) Essential readings in problem based learning. Purdue University Press, West Lafayetter

Yang CWT, Ouellet E, Lagally ET (2010) Using inexpensive jell$\mathrm{O}$ chips for hands-on microfluidics education. Anal Chem 82(13):5408-5414 